\title{
A phenothiazine derivative in the treatment of spasticity
}

\author{
DAVID BURKE, CHARLES HAMMOND, NEVELL SKUSE, \\ AND RICHARD F. JONES \\ From the Division of Neurology and the Department of Rehabilitation Medicine, \\ The Prince Henry Hospital, Little Bay, N.S.W. 2036, Australia
}

SYNOPSIS The efficacy of a selective fusimotor suppressant, the phenothiazine $( \pm)-10-(3-$ dimethylamino-2-methylpropyl)-2-valeroylphenothiazine, has been assessed in a double-blind crossover trial in eight patients suffering from cerebral spasticity and one patient suffering from spinal spasticity. Dosage was $40 \mathrm{mg}$ daily. Independent clinical and electromyographic methods of assessment were used. The active agent produced a small but significant reduction in spasticity, although this was of clinical value in only a few patients. There were few side-effects. It is recommended that further studies using higher dosages be undertaken.

Neurological thought about spasticity has been dominated by the view that hyperactivity in the fusimotor/muscle spindle system is, if not the major pathophysiological factor, at least a significant contributing factor to the increased tone of spasticity (Rushworth, 1960, 1964; Jansen, 1962). This theory has not met with universal acceptance. Studies of muscle spindle afferent activity in spastic man (Hagbarth et al., 1973), and in monkeys with chronic lesions involving areas 4 and 6 of the cerebral cortex (Gilman et al., 1974) have failed to demonstrate the heightened responsiveness that would result from increased fusimotor drive. An assessment of the efficacy of drugs which block fusimotor function is therefore of interest not only from a therapeutic viewpoint, but also because of the light such an agent may shed on the pathophysiological mechanisms of spasticity.

Phenothiazines have long been known to be capable of abolishing the decerebrate rigidity produced by transection of the mid-brain, a form of rigidity critically dependent on an intact fusimotor system (Henatsch and Ingvar, 1956). Recently phenothiazines have been shown to reduce the firing rate of both static and dynamic fusimotor neurones in doses comparable with those which abolish decerebrate rigidity (Max(Accepted 30 November 1974.) well and Rhodes, 1970; Maxwell and Sumpter, $1972,1974)$ and it has been suggested that the site of action is the brain-stem reticular formation, on a bulbospinal noradrenergic pathway (Maxwell and Sumpter, 1972, 1974). The clinical usefulness of some phenothiazines, such as chlorpromazine, has been limited by soporific effects, but with other derivatives it has proved possible to separate sedative activity from the depressant action on the fusimotor system (Keary and Maxwell, 1967). One such derivative, dimethothiazine, has demonstrated some beneficial activity in spastic man (Matthews et al., 1972), and in childhood cerebral palsy (Griffiths and Bowie, 1973), but phototoxicity, and other problems limit its usefulness. A more recent derivative, ( \pm )-10-(3-dimethylamino-2-methylpropyl)-2-valeroylphenothiazine; also known as M \& B 18706, has proved to be a more potent and potentially less toxic agent (Maxwell and Sumpter, 1972, 1974; Maxwell et al., 1974). Like other phenothiazines, this drug appears to have selective action on the fusimotor system, an action that can fully explain its effects on decerebrate rigidity (Maxwell et al., 1974). Unlike baclofen, an agent of established efficacy in spinal spasticity, it has no significant effect on standard segmental spinal reflexes (Maxwell et al., 1974). 
This phenothiazine derivative has been subjected to double-blind clinical evaluation in a small group of patients with predominantly cerebral spasticity, the clinical findings being complemented by an objective assessment based on the electromyographic analysis described by Jones et al. (1970), and Ashby et al. (1972). The encouraging results of the trial suggest that further evaluation is warranted.

\section{METHODS}

Nine patients were studied. Spasticity resulted from cerebral lesions in eight patients, five of whom suffered residual spastic hemiparesis after thrombotic or haemorrhagic strokes, and two of whom were hemiparetic after trauma. The eighth patient was hemiparetic due to an undiagnosed cortical lesion, and the remaining patient was quadriplegic due to a Klippel-Feil anomaly which had resulted in cervical spine cord compression. Of the eight patients with cerebral spasticity, the 'dystonic' posture of flexed and adducted upper limb and extended lower limb was present in four. The patient with spinal spasticity was previously maintained on baclofen which, although reducing his severe spasticity, did not control it adequately. At the time of the trial the disability of each patient was stable.

A double-blind crossover study was carried out to compare the efficacy of M \& B 18706 with that of a placebo. All sedatives, tranquillizers, and muscle relaxants in use before the trial were suspended at least one week before the study commenced. Each treatment period was of two weeks' duration. Patients were assigned randomly to one treatment group, crossing over to the other after two weeks. The active agent was administered in tablets of 10 $\mathrm{mg}$, the daily dosage being $20 \mathrm{mg}$ in two divided doses for the first seven days followed by $40 \mathrm{mg}$ in two divided doses for the subsequent seven days. Identical placebo tablets were administered in a similar manner.

Clinical assessments were carried out regularly by two of the authors on seven of the 14 days in each of the treatment periods. The clinical assessment evaluated functional changes, muscle power, muscle tone, tendon hyperreflexia, and clonus. Voluntary power was graded using the Medical Research Council scale. Muscle tone was graded from no resistance (0), through slight (1), moderate (2), and marked resistance (3) to complete rigidity of the limb. Tendon reflexes were recorded as 0 (absent), 1 (reduced), 2 (normal), 3 (increased), and 4 (markedly increased). The duration of muscle clonus was recorded in seconds, longer than 30 seconds being regarded as maximal.
The electromyographic (EMG) assessment was performed by two of the authors on the 13th or 14th day of each treatment period. This assessment was carried out completely independently of the clinical evaluation, there being no collusion between authors until the completion of the study. Full details of the electromyographic methods have been given in previous trials (Jones et al., 1970; Ashby et al., 1972). The reflex EMG responses of the quadriceps and hamstrings muscle groups were recorded by surface electrodes in standardized positions while the muscles were stretched passively at different rates by manual movements of the knee. Direct EMG, integrated EMG, knee joint position, and velocity of stretch were recorded for each muscle group.

For each patient the dynamic stretch reflex was then represented by a scatter diagram produced by plotting the velocity of the stretching movement against the amplitude of the resulting integrated EMG response. Linear regression analysis was performed for those movements which produced EMG, the correlation coefficient of the regression line being considered significant only if it exceeded 0.6. Nonsignificant data were excluded from further calculations. The intercept of the regression line on the velocity axis (the threshold velocity) was used for statistical comparison of the active and placebo

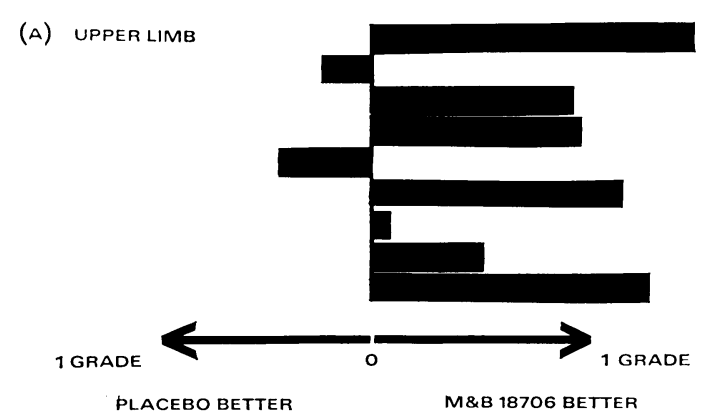

(B) LOWER LIMB

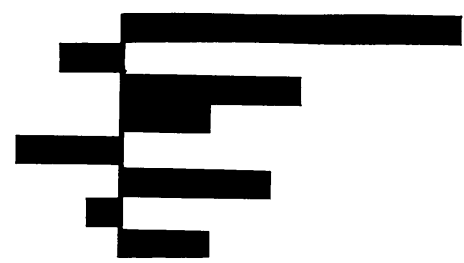

FIG. 1 Clinical assessment of changes in muscle tone. These changes were not great, being rarely more than one grade. For each patient, the average difference between the assessments when on placebo and active agent is shown. The patients are shown from top to bottom (1-9) as they occurred in the trial. 
treatments. The EMG activity produced at the velocity of $300^{\circ}$ /second was derived from the regression equation, and was used as a further point of comparison for statistical purposes. In previous trials the velocity of $200 \%$ s was used for this purpose but on this occasion it was found that the threshold velocity was occasionally close to $200 \%$, so that a small increase in threshold velocity produced a disproportionately large percentage change in EMG at $200^{\circ} / \mathrm{s}$, and thus provided a misleading overestimate of the changes produced by the active agent. The significance of differences in these two parameters between the active and placebo treatments was determined using the two-tailed Student's test.

Patients were specifically questioned to determine side-effects of medication, with particular attention to drowsiness, postural hypotension, and phototoxicity. Other phenothiazine side-effects were looked for on physical examination. At the end of each trial period, full blood count, reticulocyte count, erythrocyte sedimentation rate, serum electrolytes and creatinine, blood urea, liver function tests, electrocardiogram, and electroencephalogram were carried out.

\section{RESULTS}

CLINICAL ASSESSMENT The reduction in muscle tone on active therapy was greater than that on placebo therapy in seven of the nine patients as assessed in the upper limb, and in five of eight patients in the lower limb, the ninth patient having had no evidence of increased tone in the lower limbs in pre-trial assessments (Fig. 1). In two patients (nos 2 and 5), in whom the placebo response appeared greater than that on $\mathrm{M} \& \mathrm{~B}$ 18706, spasticity was mild, the changes were small, and the active agent had been administered in the first treatment period. The slight improvement achieved by the end of this treatment period appeared to be quite well maintained during the subsequent placebo phase, there being no intervening period during which no tablets were administered. Random allocation of medication resulted in only three patients commencing active therapy in the first treatment period. The third patient, who had more severe spasticity, also noticed some continuation of improvement into the subsequent placebo phase (greater ease of walking due to lessened 'stiffness'), but to objective examination muscle tone reverted to the pre-trial level. Thus, compared with pre-trial assessments, spasticity decreased

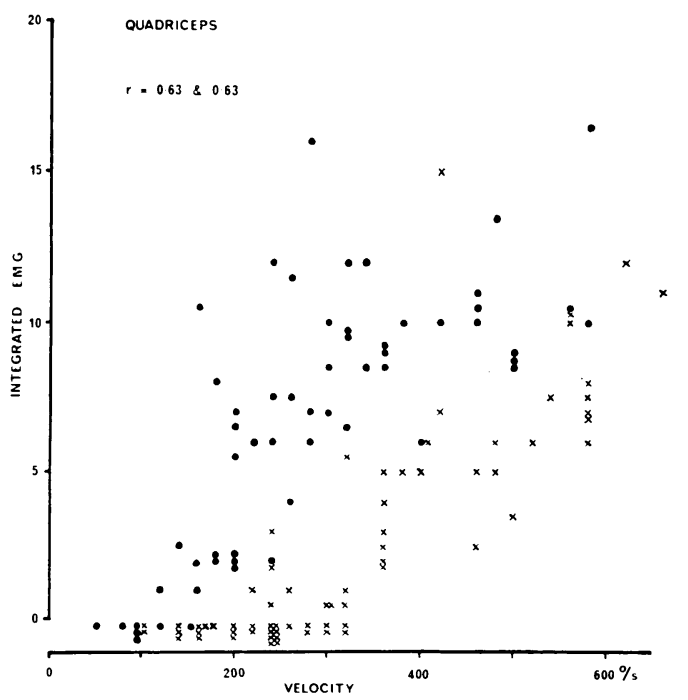

(a)

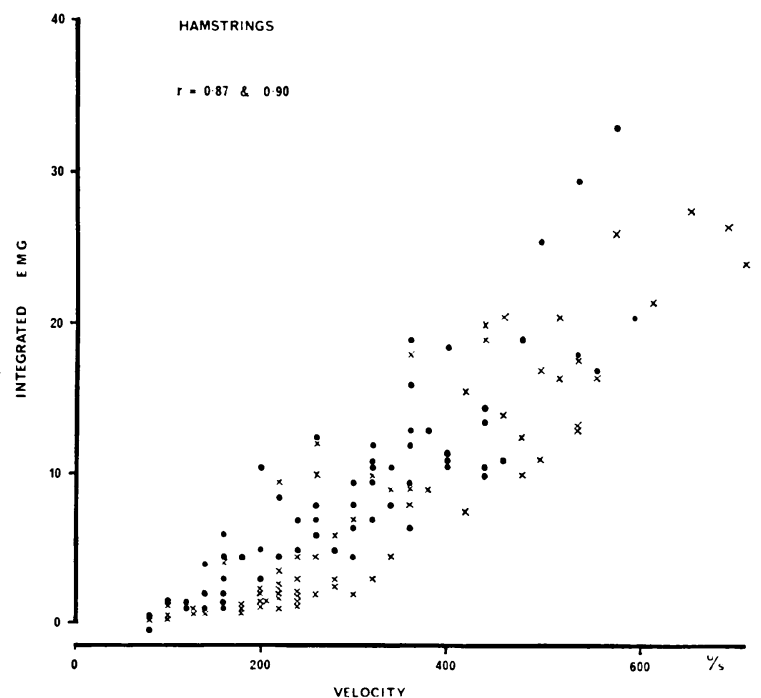

(b)

FIG. 2 The EMG: velocity relationship. Scatter diagrams obtained from (a) the quadriceps and (b) the hamstrings of patient no. 6 , placebo treatment being represented by dots and active treatment by crosses. The $r$ values represent the correlation coefficients of the linear regression lines obtained in each muscle testing. The quadriceps correlation coefficients (0.63 and 0.63) were the lowest values accepted as being sufficiently linear. 
during the trial in eight of the nine patients, each to a variable extent, although these benefits could not be confidently attributed to the active agent alone.

During treatment with the active agent, power of intrinsic muscles of the hand (finger adductors) improved in three patients, two of whom had no obvious active finger adduction before treatment. This unexpected finding presumably resulted from greater ease of movement because of a reduction in spastic stiffness. There was no uniform or sustained change in tendon reflexes or clonus. Such a change might be expected with an agent which acts on the fusimotor system; the absence of such an effect could result from difficulties of accurate clinical assessment.

Treatment with M \& B 18706 resulted in functional improvement in four patients (two slight, one moderate, one marked). Three patients noted no functional difference, while two patients (numbers 6 and 8 ) felt they were worse on the active agent due to side-effects, and to greater clumsiness resulting from a definite reduction in spasticity. After completion of the trial, three of the five patients who failed to gain functional benefit have since noticed a slight deterioration, and have expressed a preference to remain on the active agent.

ELECTROMYOGRAPHIC ASSESSMENT Inspection of the scatter diagrams of integrated EMG plotted against velocity of the stretching movement showed that for each patient a smaller reflex EMG response was produced during active treatment than during placebo treatment. The differences were small, and not of the same degree in the hamstrings and the quadriceps muscles (Fig. 2). In three subjects no appreciable change in the EMG velocity relationship resulted from treatment in one muscle group, but active treatment resulted in a slight reduction in the EMG response in the other muscle group. The remaining six subjects had a minor improvement in one muscle group accompanied by moderate to marked changes in the other.

Statistical analysis was performed for the data from eight patients, that from the ninth being rejected as unreliable because the patient was tense and unable to relax during the testing and correlation coefficients were low. Data on the quadriceps muscle of a second patient were not
TABLE 1

CORRELATION COEFFICIENTS

\begin{tabular}{llccc}
\hline Muscle group & Treatment & Number & Range & Mean \\
\hline Quadriceps & Placebo & 7 & $0.63-0.91$ & 0.78 \\
& Active & 7 & $0.63-0.94$ & 0.84 \\
Hamstrings & Placebo & 7 & $0.71-0.91$ & 0.84 \\
& Active & 7 & $0.65-0.94$ & 0.81 \\
\hline
\end{tabular}

TABLE 2

THRESHOLD VELOCITY INCREASE

\begin{tabular}{lcccc}
\hline Muscle group & Number & Mean & Range & Probability \\
\hline Quadriceps & 7 & 43.6 & -26.4 to +160.4 & NS \\
Hamstrings & 7 & 34.9 & -47.0 to +107 & NS \\
Pooled & 14 & 39.3 & -47.0 to +160.4 & $<0.02$ \\
\end{tabular}

TABLE 3

EMG DECREASE AT $300^{\circ} / \mathrm{s}$

\begin{tabular}{lrccc}
\hline Muscle group & Number & $\begin{array}{c}\text { Mean } \\
\text { (units) }\end{array}$ & Range & Probability \\
\hline Quadriceps & 7 & 4.52 & -1.86 to +10.05 & $<0.02$ \\
Hamstrings & 7 & 2.11 & -0.91 to +5.70 & $<0.05$ \\
Pooled & 14 & 3.31 & -1.86 to +10.05 & $<0.005$ \\
\hline
\end{tabular}

significantly linear (low correlation coefficients), and the data on the hamstrings of a third patient were unreliable due to technical problems. Although excluded from further analysis, inspection of these data suggested that active treatment was beneficial in each case. Thus in the eight subjects further analysis was performed for the quadriceps in seven and for the hamstrings in seven. The correlation coefficients for these regression equations are detailed in Table 1.

The increases in threshold velocity produced by the active agent are shown in Table 2 . When each muscle group was considered separately the increase was not statistically significant, possibly due to the small sample sizes. On pooling the data from the two muscle groups to provide a larger sample, the mean increase in threshold velocity of $39.3^{\circ} / \mathrm{s}$ was quite significant $(P<0.02)$. The decrease in EMG activity produced at $300^{\circ} / \mathrm{s}$ resulting from active treatment (Table 3 ) 
was significant in each muscle group when considered separately, and was highly significant $(\mathrm{P}<0.005)$ when the data from the two muscle groups were pooled.

Although the changes produced by treatment were small, they were largely in the same direction, and consequently they achieved statistical significance, a significance that has resulted not from the magnitude but from the consistency of the change. While it may be concluded that a reduction in spasticity has been demonstrated, it cannot be concluded that worthwhile clinical improvement accompanied this reduction. Certainly the magnitude of the electromyographic change was such that in at least two patients significant clinical improvement might be expected. This is particularly so in the sole patient with spinal spasticity. During placebo treatment the reflex activity of this patient's hamstrings was so intense that stretching movements produced prolonged EMG activity which subsided very slowly after cessation of movement. This 'static' stretch reflex response was virtually abolished by the active treatment.

SIDE-EFFECTS The administration of $\mathrm{M} \& \mathrm{~B}$ 18706 did not alter the biochemical screening tests except for several isolated instances of elevation of serum alkaline phosphatase. On clinical examination no side-effects were apparent.

Minor subjective symptoms elicited on interrogation included dry mouth and drowsiness. These occurred during the active treatment phase and were transient. Patients nos 2 and 5 developed an influenza-like illness during the trial, patient 2 being mildly affected for two days early in the first week of the placebo treatment phase, and patient 5 being more severely affected during the active treatment phase. It is possible that this intercurrent illness may have contributed to the placebo response in patient 5 . No patient ceased the drug because of side-effects.

\section{DISCUSSION}

The assessment of the efficacy of medication for the relief of spasticity must rely on clinical evaluation, subjective feelings of improvement, changes in degree of independence, and changes in the activities of daily living. The additional value of the EMG assessment lies in its objectivity and in its ability to discriminate early changes which may not yet be clinically obvious. In the dosage used, clinical improvement could be found in most patients, although this was small. Nevertheless, the phenothiazine, M \& B 18706, has proved of definite value in two patients. Interestingly, one of the patients to benefit most suffered from spinal spasticity, the degree of improvement at least equalling that of the patient's previous medication, baclofen. Significantly, the 'dystonic' posture that may accompany cerebral spasticity was not altered by treatment.

A number of therapeutic conclusions can be drawn from these results. In the dosage used, significant clinical benefit, sufficient to justify the use of the medication, may occur in only a few patients, although a reduction in spasticity may be anticipated in most. Since the drug was well tolerated in a dosage which produced a slight reduction in spasticity in most patients, it might be anticipated that higher dosages would result in greater benefits, and such dosages are recommended for future trials. Usage of the drug should be extended to patients with spinal spasticity, including multiple sclerosis, although it is probable that benefit would occur only in those patients with incomplete spinal lesions. In patients with severe spinal spasticity, a combination of agents with different mechanisms of action should prove synergistic, such that the combination of the phenothiazine, which suppresses fusimotor activity, with baclofen, which suppresses segmental reflex transmission, should produce benefits greater than either drug alone.

The conclusions that can be drawn from this trial concerning the pathophysiology of spasticity are limited. The reduction in spasticity produced by the drug demonstrates a role for the fusimotor system in spasticity, but the small size of this reduction does not support the preeminence of this role. However, the dosages used may not have been optimal. Clearly, definite conclusions must await continuing evaluation.

The authors are grateful to Miss M. Mackie for random allocation of medication, to Dr David Manohar for assistance with clinical evaluations, to Professor James W. Lance for support and help in the preparation of this paper, and to May \& Baker Ltd for supplies of the active agent and placebo. 


\section{REFERENCES}

Ashby, P., Burke, D., Rao, S., and Jones, R. F. (1972). Assessment of cyclobenzaprine in the treatment of spasticity. Journal of Neurology, Neurosurgery, and Psychiatry, 35, 599-605.

Gilman, S., Lieberman, J. S., and Marco, L. A. (1974). Spinal mechanisms underlying the effects of unilateral ablation of areas 4 and 6 in monkeys. Brain, 97, 49-64.

Griffiths, M. I., and Bowie, E. M. (1973). The use of dimethothiazine in the treatment of childhood cerebral palsy. Developmental Medicine and Child Neurology, 15, 25-32.

Hagbarth, K.-E., Wallin, G., and Löfstedt, L. (1973). Muscle spindle responses to stretch in normal and spastic subjects. Scandinavian Journal of Rehabilitation Medicine, 5, 156-159.

Henatsch, H. D., and Ingvar, D. H. (1956). Chorpromazin und Spastizität. Eine experimentelle elektrophysiologische Untersuchung. Archiv für Psychiatrie und Nervenkrankheiten, 195, 77-93.

Jansen, J. K. S. (1962). Spasticity-functional aspects. Acta Neurologica Scandinavica, 38, Suppl. 3, 41-51.

Jones, R. F., Burke, D., Marosszeky, J. E., and Gillies, J. D. (1970). A new agent for the control of spasticity. Journal of Neurology, Neurosurgery, and Psychiatry, 33, 464-468.

Keary, E. M., and Maxwell, D. R. (1967). A comparison of the effects of chlorpromazine and some related phenothiazines in reducing the rigidity of the decerebrate cat and in some other central actions. British Journal of Pharmacology and Chemotherapy, 30, 400-416.

Matthews, W. B., Rushworth, G., and Wakefield, G. S. (1972). Dimethothiazine in spasticity. Acta Neurologica Scandinavica, 48, 635-644.

Maxwell, D. R., Read, M. A., and Sumpter, E. A. (1974). Pharmacology of M \& B 18706, a drug which selectively reduces decerebrate rigidity. British Journal of Pharmacology, 50, 35-45.

Maxwell, D. R., and Rhodes, K. F. (1970). Studies of the effects of dimethothiazine on muscle spindle activity in the decerebrate cat. Journal of Physiology, 208, 29-30P.

Maxwell, D. R., and Sumpter, E. A. (1972). Noradrenergic receptors and the control of fusimotor activity. Journal of Physiology, 222, 173-175P.

Maxwell, D. R., and Sumpter, E. A. (1974). A comparison of the actions of some drugs on decerebrate rigidity, muscle spindle activity, and $\alpha$-adrenoceptors. British Journal of Pharmacology, 50, 355-363.

Rushworth, G. (1960). Spasticity and rigidity: an experimental study and review. Journal of Neurology, Neurosurgery, and Psychiatry, 23, 99-118.

Rushworth, G. (1964). Some aspects of the pathophysiology of spasticity and rigidity. Clinical Pharmacology and Therapeutics, 5, 828-836. 